\title{
8 Green Aspects of Miniaturized Sample Preparation Techniques
}

\author{
Adam Kloskowskia*, tukasz Marcinkowskia and Jacek Namieśnik ${ }^{\mathrm{b}}$ \\ ${ }^{a}$ Gdansk University of Technology, Faculty of Chemistry, Department of Physical Chem- \\ istry, Narutowicza Str.11/12, Gdansk 80-233, Poland. \\ ${ }^{b}$ Gdansk University of Technology, Faculty of Chemistry, Department of Analytical \\ Chemistry, Narutowicza Str.11/12, Gdansk 80-233, Poland. \\ *e-mail: adam.kloskowski@pg.gda.pl
}

\subsection{Introduction}

Correct monitoring and control of the state of the environment and scientific research on the subject of environmental protection often require that determinations of compounds present at very low concentrations be made in samples characterized by a matrix of complex composition (e.g., biological samples, water and soil samples). Moreover, due to the limited number of analytical techniques which are sufficiently sensitive to conduct direct determinations of trace components, it is necessary to employ analytical procedures that involve an analyte isolation and enrichment step prior to final analyte quantification. Technical and methodological solutions described in previous chapters allow for reduced dimensions of equipment and the amount of material used at the sample preparation stage prior to analysis. Therein, the reader will find a detailed description of specific techniques and their associated analytical methods, including their range of application for isolating certain types of analytes from various kinds of samples. The aim of this chapter is to evaluate pro-ecological effects resulting from the miniaturization of sample preparation techniques. Although the elaboration of most of the aforementioned solutions was dictated by very different demands, e.g., cost reduction, ease of application and the ability to create hyphenated analytical systems, the environmental effects are also considered to be a key benefit to their development.

A discussion of the relationship between the miniaturization of analyte isolation and enrichment techniques and the requirements posed by green analytical techniques should begin by reminding the reader of the fundamental targets that guide green analytical chemistry (GAC). At present, it is commonly accepted that the development of analytical techniques should proceed by following the priority issues:

1. discontinuation of or significant reduction in the use of environmentally-harmful chemical compounds in analytical procedures, with a special emphasis on organic solvents

2. limiting the emission of vapors and gases

(cc) BY-NC-ND (c) 2014 Adam Kloskowski, tukasz Marcinkowski and Jacek Namieśnik This work is licensed under the Creative Commons Attribution-NonCommercial-NoDerivs 3.0 License. 


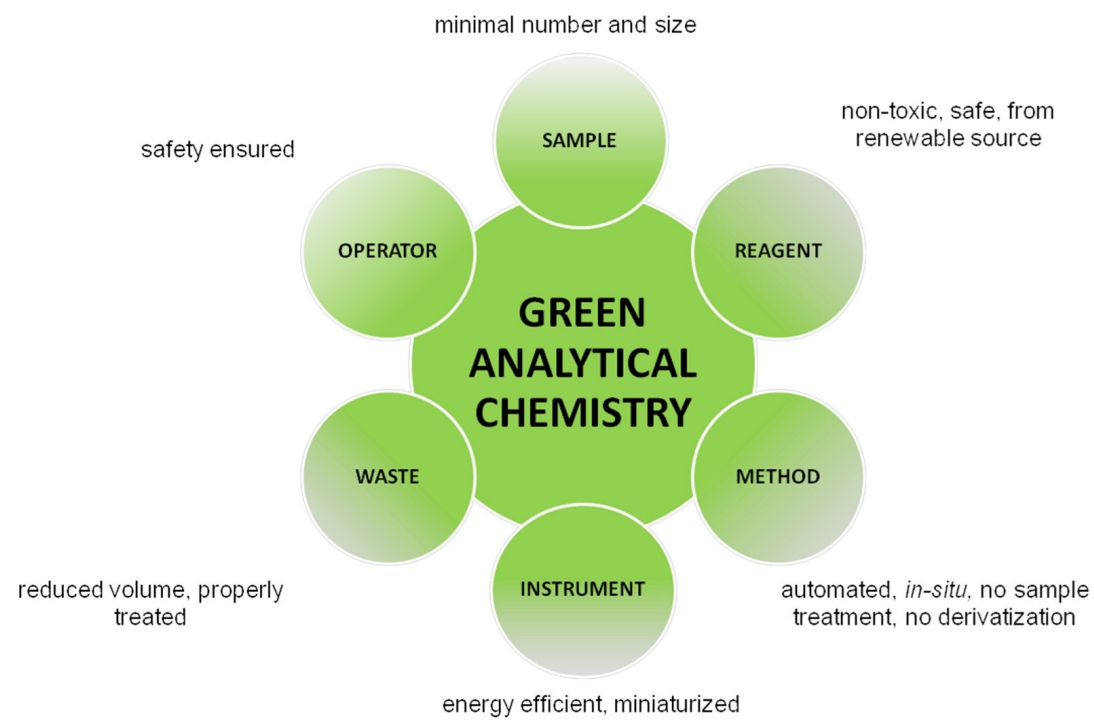

Figure $\mathbf{8 . 1}$ Important components of analytical procedures in relation to the principles of GAC.

3. limiting the amount of liquid and solid wastes generated by analytical laboratories

4. introduction of solutions into analytical practice which would allow the application of environmentally-friendly solvents such as water and supercritical $\mathrm{CO}_{2}$

5. reduction of energy- and labor-intensive analytical procedures

6. possible automation

Important components of analytical procedures in relation to the principles of GAC are shown in Figure 8.1.

First of all, it should be mentioned that the fulfillment of some of the aforementioned priorities is an obvious consequence of the miniaturization of sample preparation techniques. Undoubtedly, a reduction in the use of organic solvents, vapor emissions, and the amount of generated wastes will accompany miniaturization. A similar relationship can be found between the miniaturization and lowered energy intensity, for example, via decreasing energy used per unit when thermostating a sample or by reducing the amount of solvent being evaporated in the process of analyte condensation. On the other hand, the same aims can be and often are achieved not by scaling down, but rather by choosing appropriate materials used in microextraction techniques as well as the procedures for their application. As an example might serve the application of polydimethylsiloxane (PDMS). This polymer is applied as a sorbent, solely, in microextraction techniques. Its properties allow multiple use and thermal desorption of analytes, thus eliminating the need of using organic solvents. It should 
also be mentioned that the sample preparation techniques are not easily classified according to their green features because in many cases the application of one technological solution allows for achieving multiple aims. However, other goals, such as automation of the procedure often require additional efforts. Solid-phase microextraction (SPME) is a flagship example of GAC because the use of polymers as a sorption phase and the release of analytes via thermal desorption allows for a complete elimination of organic solvents from the analytical procedure, and therefore points 1-3 are fulfilled. In the case of thermally sensitive analytes, the same technique requires that analytes are extracted from the fiber with solvents, thus reducing the green character of the procedure. A similar situation is related to the possible automation of the analyte isolation step by using SPME. Technically, such a solution was achieved only a few years ago when the specialized fiber holder and automated sampler were constructed. The automation of analytical procedures is relatively simple only in the case of thermal desorption of analytes where the SPME fiber is introduced directly into the GC injector, whereas in the case of desorption with solvents, more sophisticated devices are used (Hutchinson et al., 2007).

Considering the above information, we will discuss microextraction techniques for sample preparation with regard to the materials used, design solutions and possible automation. Finally, the application of chemometric tools in microextraction techniques is briefly described.

\subsection{Reduction of the Amount of Organic Solvents Used}

To begin the process of reducing the amount of organic solvent used in an analysis, a reference point has to be chosen. This is usually the technique that is most commonly used in practice, which for many processes is the sample preparation step based on liquid-liquid extraction (LLE) with organic solvents. LLE is one of the oldest extraction techniques, mostly applied to aqueous samples that are characterized by a matrix of simple to complex composition. In general, the technique involves mixing the sample with a fresh aliquot of solvent, leading to the separation of analytes depending on their affinity for the two components of the system (sample phase and extractant). The use of solvents with increasing polarity creates an opportunity to obtain different fractions which are enriched in various analyte groups. Moreover, another step in the procedure is solvent evaporation aimed at condensing the dissolved analytes. Multi-step procedures are highly time- and labor-intensive and, as a consequence, result in longer exposure of the laboratory personnel to harmful vapors of chemical reagents, particularly of organic solvents. Also, a relatively large number of operations performed on the sample increases the risk of analyte loss and sample contamination. From the point of view of green chemistry priorities, in this type of procedure the only requirement which can be fulfilled is the application of environmentally friendly solvents, e.g., water and supercritical $\mathrm{CO}_{2}$. All design solu- 
tions lead, in a natural way, to the miniaturization of the dimensions of equipment used for isolating analytes and, at the same time, to decreased use of organic solvents. As a consequence, the amount of waste produced and the emissions of vapors and gases are diminished. Nevertheless, it should be mentioned that the same phenomenon is used for isolating analytes (separation based on partitioning between phases) by liquid-phase microextraction (LPME) techniques and classical LLE techniques. Therefore, LPME is not always a practical alternative to typical LLE techniques, for example, in situations when high recovery instead of high enrichment of analytes is required. In such a scenario, due to the limited volume of extractant, the competitiveness of LPME techniques is based on the extractant's physical properties, in particular on its affinity for target analytes. In this context, miniaturization of extraction techniques allows a search for alternative extractants among rare and/ or expensive materials.

Single-drop microextraction (SDME) is one of the most popular techniques in which the use of solvent has been significantly reduced to only one drop with a volume of $1-8 \mu \mathrm{L}$ compared to classical LLE. SDME was developed in 1996 under the name Solvent Extraction in a Microdrop (Liu \& Dasgupta, 1996) or Solvent Microextraction into a Single Drop (Jeannot \& Cantwell, 1996). In this technique, the extraction process occurs via dissolution of analytes in a drop of solvent suspended at the end of microsyringe needle. The needle is immersed in the sample or sample headspace (HS-SDME mode) (Tankeviciute et al., 2001). In the case of HS-SDME, the choice of solvent is wider because its solubility in the sample is of no significance. In order to improve the stability of the suspended drop, needles with specifically shaped ends and made of various materials are used (Ye et al., 2007; Batlle et al., 2008; Sharma et al., 2011).

In some cases, it is also possible to reduce the sample volume. The drop-todrop SDME technique (DD-SDME) enables fast extraction and eliminates the sample mixing because the analytes are extracted into a drop of solvent from the sample of a very small volume, usually no more than $10 \mu \mathrm{L}$ (Wu et al., 2006). After the extraction of analytes, the drop of liquid sorbent is drawn into the syringe with the plunger and then injected into the dosing device of measuring instrument. The extraction of analytes by SDME techniques is characterized by short extraction time, low cost, simplicity of operation, and no need for complicated equipment.

A further reduction of the amount of solvents used has been achieved in the labon-a-chip version known as droplet-membrane-droplet-LPME, in which the analytes are extracted through a liquid membrane into a few microliters of acceptor phase (Sikanen et al., 2010). The chip used in droplet-membrane-droplet-LPME technique is made of appropriately molded aluminum foil. The sample phase and the extractant phase $(10 \mu \mathrm{L})$ are separated by microporous polypropylene membrane that had been impregnated with a solvent. A small amount of sample $(10-15 \mu \mathrm{L})$ is placed onto the membrane. After the extraction, the acceptor phase is collected with a microsyringe and then introduced into an analytical instrument. 
To make SDME compatible with gas chromatography (GC), mainly organic solvents (1-octanol, toluene, dodecanol, undecanol) or chloroorganic solvents (dichloromethane, chloroform, carbon tetrachloride, trichloroethylene, dichloroethylene) are used as extractants (Romero et al., 2007). However, the application of the latter is being discontinued due to their toxicity. Information about the application of other less traditional solvents, such as aqueous solutions of $\beta$-cyclodextrin, can be found in the literature (Wu et al., 2008).

The modifications of classical SDME are also worth mentioning. The directly suspended droplet microextraction technique (DSDME) involves the placement of a drop of water-immiscible solvent directly on the sample surface. After the extraction, solvent is collected from the sample surface with a microsyringe (Yangcheng et al., 2006; Pena-Pereira et al., 2012). From the point of view of green principles, the main disadvantage of this solution is that the transfer of extract to the next steps of analytical procedure cannot be automated. Solidified floating organic drop microextraction technique (SFODME) is also an interesting option (Khalili Zanjani et al., 2007). In this technique, a drop of extractant immiscible with the sample matrix is placed on the sample surface and then stirred at constant temperature. After the extraction, the vessel containing the sample and extractant is cooled until the extraction medium solidifies. Next, the extractant phase with the absorbed analytes is transferred to another vessel where it melts. Then the extraction medium is dosed into a measuring device (Sobhi et al., 2008). As in the case of DSDME, the GAC limitation of SFODME is that the transfer of sample into a measuring device cannot be automated.

Hollow fiber liquid-phase microextraction techniques (HF-LPME) are another solution that allows a reduction in the amount of solvents used (Pedersen-Bjergaard \& Rasmmussen, 1999). In this group of techniques, a small volume of liquid extraction medium (a few $\mu \mathrm{L}$ ) is contained within the lumen of porous polypropylene fiber which is attached to the end of microsyringe needle and immersed in the sample (Zhu et al., 2001). Extraction by HF-LPME technique can be conducted either in a two-phase system, where the wall and lumen of the fiber are filled with the same solvent, or in a three-phase system in which the fiber wall is impregnated with different solvent than that present in the fiber lumen. A wide choice of available fibers (Kosaraju \& Sirkar, 2007) and solvents enables the achievement of high extraction selectivity for different analytes, and allows the application of HF-LPME for sampling analytes from samples which are contaminated or have complex matrices.

As mentioned previously, the limitation of microextraction techniques based on the partitioning mechanism is the small amount of extractant used. As a consequence, the high affinity of the extractant for the analytes is much more significant for the quality of the obtained results in comparison to classical LLE. For example, in order to increase the extraction efficiency, multiwall carbon nanotubes (MWCNTs) dispersed in a specific extractant can be used. The hollow fiber solid-liquid phase microextraction (HF-SLPME) technique ensures high selectivity and good efficiency of analyte extraction even when the volume of sorption phase is very small (Es'haghi 
et al., 2010). Extraction can also take place in a two-phase system in which carbon nanotubes suspended in organic solvent fill the lumen of the hollow fiber (Es'haghi et al., 2011). A similar system has been designed for solvent bar microextraction (SBME) technique (Jiang \& Lee, 2004), where the extractant is immobilized inside the pores of a polypropylene fiber. Both ends of the fiber are closed and the fiber is either filled with the extractant (liquid-liquid system), or with an acceptor phase that is different than the extractant (liquid-liquid-liquid system) (Yu et al., 2008). After the extraction, the acceptor phase is collected from the fiber lumen with a microsyringe and injected into the GC or high performance liquid chromatography (HPLC) device. In relation to HF-LPME, it is noteworthy that automation of analytical procedure (Ouyang et al., 2006) and concurrent sampling of analytes from multiple samples is possible, which is important with regard to the green character of this technique. In the case of solvent cooling assisted dynamic hollow-fiber-supported headspace liquid phase microextraction (SC-DHF-HS-LPME), the extractant is pumped through the porous polymeric fiber which has been cooled to $-1^{\circ} \mathrm{C}$, to lower the solvent vapor pressure and therefore the solvent loss (Huang et al., 2007).

Another group of solvent microextration techniques is based on the dispersion of a small amount of solvent (up to $200 \mu \mathrm{L}$ ) in the investigated sample. In the basic variant of dispersive liquid-liquid microextraction (DLLME), the dispersed droplets of extractant are generated by using the third liquid phase, which does not mix with the sample or the extractant and acts as a dispersant (Rezaee et al., 2006). After the extraction, samples are shaken and centrifuged to separate the extraction phases, which is later injected into the appropriate analytical instrument. The presence of a dispersant in the system (usually methanol, acetone or acetonitrile in the amount of $0.5-2 \mu \mathrm{L}$ ) may lower the value of the analyte-specific partition coefficient during the partitioning of analytes between the sample phase and extraction phase. This is undesirable from the point of view of green chemistry, therefore, dispersion by means of ultrasound (ultrasound-assisted emulsification-microextraction (USAEME) or ultrasound-assisted dispersive liquid-liquid microextraction (US-DLLME) (Regueiro et al., 2008; Fontana et al., 2009) or vortex mixing (vortex-assisted liquid-liquid microextraction (VALLME) is often applied (Jia et al., 2010; Yiantzi et al., 2010). Moreover, generation of very small droplets of extractant results in a significant increase in the interphase surface of both immiscible liquids, which speeds up the transfer of analytes to the extractant and consequently decreases the time necessary for isolating the analytes. Another solution which eliminates the use of dispersing solvents from the procedure is the application of surfactant solutions, often in combination with ultrasound treatment. In this way, techniques such as surfactant-assisted dispersive liquid-liquid microextraction (SADLLME) (Saraji \& Bidgoli, 2010), ion pair-based surfactant-assisted microextraction (IP-SAME) (Moradi et al., 2011), ultrasound-assisted surfactant-enhanced emulsification microextraction (UASEME) (Wu et al., 2010) and vortex-assisted surfactantenhanced-emulsification liquid-liquid microextraction (VSLLME) (Yang et al., 2011) were developed. Another aspect of modified DLLME techniques demonstrates that the 
principles of GAC are often realized by making a compromise on selected targets. It turns out that the previously criticized use of dispersant may result, under specific conditions, in the demulsification of the extractant. Guo and Lee (2011b) described a procedure where a mixture of hexane and acetone was added, leading to the creation of an emulsion. After addition of the next portion of acetone, demulsification occurred, causing separation of the extractant from the sample phase. Separated extractant could easily be retrieved with a syringe, so omission of certain steps of the analytical procedure, such as mixing, emulsification in an ultrasonic bath or freezing out the extraction phase was possible. As a consequence, the number of operations that involve sample handling decreases, which usually improves the quality of the results. It is an individual decision which option is considered more valuable in a given situation with regard to how green the whole analytical procedure should be. Demulsification is conducted after the extraction step via the addition of another portion of dispersant, which acts as a demulsifying agent. The techniques based on demulsification can be found in literature under the name of solvent-terminated dispersive liquidliquid microextraction (ST-DLLME) (H. Chen et al., 2010), and solvent demulsification dispersive liquid-liquid microextraction (SD-DLLME) (Guo \& Lee, 2011b). In both these techniques, solvents less dense than water are used which enables the collection of extract from the sample surface with a syringe.

Fully automated variants of DLLME techniques have also been developed, such as sequential injection dispersive liquid-liquid microextraction (SI-DLLME) which allows on-line injection of extracts into flame (or electrothermal) atomic absorption spectrometry atomizers (Anthemidis \& Ioannou, 2010, 2011).

A further reduction in the amount of solvents used was achieved by forcing the transport of analytes through a semipermeable membrane in an electric field. In on-chip electro membrane extraction (EME), the isolation process was conducted by using a $25 \mu \mathrm{m}$ thick porous polypropylene membrane whose pores had been impregnated with the acceptor phase. The membrane separates the microchannel ( $50 \mu \mathrm{m}$ deep and $2 \mathrm{~mm}$ wide) bored in one of the two connected plates made of poly(methyl) methacrylate (PMMA) from a well containing a small amount (a few $\mu \mathrm{L}$ ) of acceptor phase (2-nitrophenyl octyl ether (NPOE) or dodecyl acetate). The platinum wire anode is attached inside the microchannel into which sample is pumped, while the cathode is placed inside the well containing the acceptor phase. The transfer of extract to the next steps of analytical procedure is performed with a micropipette (Petersen et al., 2010).

\subsection{Green Extraction Phases for Microextraction Techniques}

In order to increase the green character of microextraction techniques, extractants which are less harmful to the environment are introduced into analytical practices. Initially, solvents such as chlorinated hydrocarbons were mainly used in DLLME 
techniques due to practical reasons. Because they are denser than water, it is possible to simply separate the phases by sample centrifugation. Recently, applications of solvents that are less dense than water as extractants in DLLME has been published (Hashemi et al., 2009). The use of this type of solvent creates a possibility to perform the extraction without a dispersant and, at times, allows for eliminating the sample centrifugation step. In most cases, the application of extractants which have lower density than water necessitated the elaboration of novel designs, for example, a narrow-necked vessel for collecting the extractant (Farajzadeh et al., 2009; Hashemi et al., 2009), a special vessel for centrifuging the emulsion in the USAEME technique (Saleh et al., 2009), a vessel which speeds up the extraction via mixing with a magnetic stirrer (Zhang et al., 2011) and a membrane system which facilitates the dosing and collection of extractant from the sample (Farajzadeh et al., 2012). Long-chain alcohols such as dodecanol and hexadecanol are often used as solvents in dispersive techniques. These solvents are commonly recognized as not very harmful to the environment. In addition, since their melting points are close to room temperature, it is possible to solidify the extractants which facilitates their quantitative collection from the sample mixture. In dispersive liquid-liquid microextraction based on the solidification of a floating organic drop (DLLME-SFOD), the extraction proceeds as in the typical DLLME. The extractant is added to and then dispersed in the analyzed sample, followed by sample centrifugation and cooling to solidify the extractant present on the top of the sample vessel. The solidified extractant is transferred to another vessel where it melts. Next, the extractant is analyzed using chromatographic techniques (Leong \& Huang, 2008; Xu et al., 2009).

Another example of the implementation of GAC principles in LPME techniques is the use of extractants which are not harmful to the environment, fo example, aqueous solutions of surfactants in cloud point extraction (CPE) (Paleologos et al., 2005). In supramolecular-based dispersive liquid-liquid microextraction (SMDLLME), coacervates and supramolecular systems serve as extractants (Jafarvand \& Shemirani, 2011). Coacervates, which are colloidal micelles (microscopic structures in the shape of droplets), self-assemble in colloidal systems while their external layer is semi-permeable and allows the analytes to permeate to the interior. Contrary to basic dispersive techniques, SM-DLLME is characterized by a short extraction time due to the dispersion of the sorption phase, and it enables the isolation of analytes with widely ranging polarity while eliminating toxic solvents and sample mixing. In the case of surfactants present in the ionized form (anionic and cationic forms), coacervates are obtained by cooling the solution below the cloud point. For nonionic surfactants, the solution should be warmed up above the cloud point. During the isolation process, poorly soluble and non-polar analytes are dissolved in the hydrophobic inner space of micelles, while polar compounds become incorporated into the external micellar structure (Yu, 2005; Madej, 2009). Because the surfactant can be adsorbed onto the capillary wall or the packing of chromatographic column, in some applications the extract is diluted before quantitative analysis (Giokas et al., 


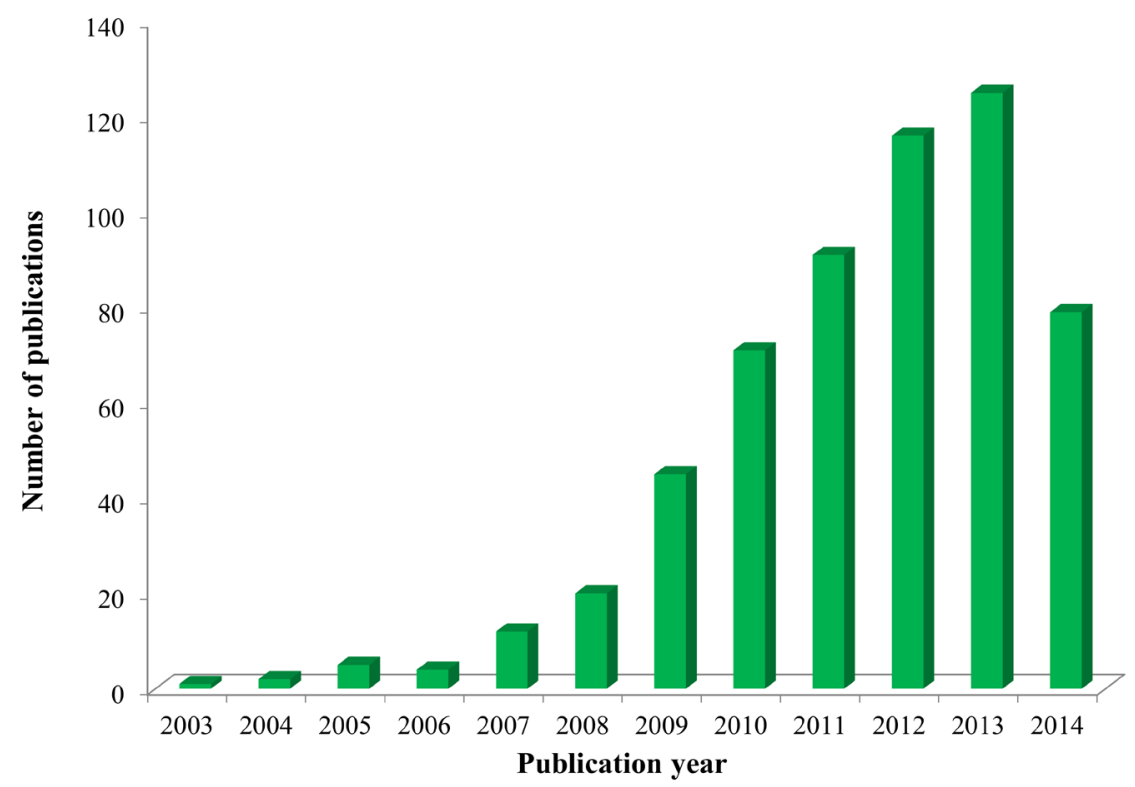

Figure 8.2 Number of publications regarding ionic liquids in microextraction techniques in the past decade.

2002), although this limits the green character of the technique. Therefore, the dual cloud point extraction (dCPE) technique was developed with the use of different surfactant (Yin, 2007). In the first step of this procedure, a surfactant solution is added to the sample containing hydrophobic analytes, which results in the formation of hydrophobic complexes with the analyte. Next, the sample is heated and centrifuged, and the complexes are extracted into the newly created phase which is rich in another surfactant. In the final step, an aqueous solution of L-cysteine is used to form a stable nd hydrophilic water soluble complexes of mercury for their subsequent transfer to aqueous phase, which is later analyzed by capillary electrophoresis (CE).

Another example of using greener substitutes is the application of ionic liquids (ILs) in microextraction techniques. The main property of ILs, which allows this group of compounds to be called green, is their very low vapor pressure and high thermal stability. Due to their high viscosity and surface tension, ILs can form a stable phase boundary which enables the extraction of analytes from the sample headspace and via direct contact with the investigated sample. As a result, one can notice a continuous growth in the the number of publications describing application of ILs in microextraction techniques, as shown in Figure 8.2. As in the case of previously described coacervates, it is noteworthy that the application of ILs results, to a certain degree, from the miniaturization of devices used in the sample preparation step. A decrease 
in the amount of extractants allowed the use of rare and expensive materials. Considering the use of ILs in microextraction techniques, one should also bear in mind that the physicochemical properties of these compounds can be controlled by properly selecting the component anions and cations.

As a result of the aforementioned properties, ILs have been used for coating fibers in SPME (Ho et al., 2011). The high viscosity of ILs allows one to obtain coatings of relatively high thickness and good stability. The first attempts at coating fibers with ILs were undertaken in 2005 (Liu et al., 2005). Unfortunately, the IL coatings produced could be used only once, and the fibers had to be coated with an IL after each use. The application of Nafion as a pre-coating substratum increased the surface wettability and therefore enabled the formation of a thicker layer of IL. The increased amount of sorption phase allowed the application of IL-coated fibers for isolating polycyclic aromatic hydrocarbons (PAHs) from aqueous samples. However, the use of Nafion did not solve the problem of repeated coating, and it was still necessary to renew the sorbent layer after each extraction (Hsieh et al., 2006). The immobilization of ILs on the fiber of the SPME device can be achieved via impregnation of silicone elastomer coatings on the fiber surface (He et al., 2009). To this end, sol-gel techniques (LópezDarias et al., 2010) or in situ cross-linking on stainless steel fibers coated with a layer of silver is used (Feng et al., 2011).

The use of ILs in SDME techniques has produced better results and the first application of an IL in SDME was reported in 2003. It was demonstrated that the extraction of PAH analytes with an IL gave better results compared to the extraction with 1-octanol for both direct sample extraction and sample headspace extraction (Liu et al., 2003). In consecutive publications, the applicability of ILs for isolating a wide spectrum of compounds including pesticides (Ye et al., 2006), phenols (Wang et al., 2010), BTEX (Aguilera-Herrador et al., 2008) and PAHs (Yao et al., 2009) was demonstrated. ILs were also successfully used to isolate inorganic compounds from food samples (Pena-Pereira et al., 2009). The instability of an IL drop and the small drop volume still remain the main problems in SDME techniques that employ ILs but new technical solutions continue to appear. In 2009, the use of an IL in dynamic liquidphase microextraction (dLPME) was described (Cruz-Vera et al., 2008). The constructed device designed for controlling the flow rate allows precise dosing of a given drop volume and enables increasing the drop volume to $50 \mu \mathrm{L}$ which translates into improved measurement sensitivity. Due to the thermal resistance of ILs, it was possible to couple SDME with GC (Aguilera-Herrador et al., 2007). After the extraction, the same syringe is used to directly introduce the droplet of ionic liquid into the GC injector where the analytes are released via thermal desorption. Only a slight modification of standard injector is necessary which involve using an inlet liner with a larger diameter to avoid the transfer of IL into the column (Chisvert et al., 2009).

The use of ILs in dispersive techniques is the natural consequence of their properties, i.e. viscosity, surface tension, and hydrophilicity/hydrophobicity. In the case of DLLME, ILs were applied in the extraction of organic compounds (aromatic amines, 
PAHs) (Pena et al., 2009) as well as metals (cadmium, lanthanides) (Martinis et al., 2008). The DLLME techniques employing ILs mainly differ in the way the extractant is dispersed. Some techniques are based on a complexing agent and surfactant, which lowers adhesive interactions between the IL and the vessel wall. On the other hand, in ionic liquid-based ultrasound-assisted dispersive liquid-liquid microextraction (ILUSA-DLLME) the dispersion effect is achieved by means of ultrasound (Arvand et al., 2012). In vortex-assisted liquid-liquid microextraction (VALLME), the IL is dispersed throughout the sample by vortex mixing (Chamsaz et al., 2013).

In in situ solvent-formation microextraction (ISFME) a hydrophobic IL and a reagent that aids in the formation of ion pairs are added simultaneously to the sample. As a result, the solution becomes cloudy due to the formation of microdroplets consisting of the IL and the aiding agent. Finally, the extract is separated by centrifugation (Baghdadi \& Shemirani, 2008). Another interesting solution, based on the broad hysteresis of ILs and their propensity to occur in the supercritical state, is coldinduced aggregation microextraction (CIAME). At first, the IL is dissolved in a warm sample. Next, the mixture is cooled in an ice bath in order to separate the extractant phase. After reaching the cloud point, the IL and the retained analytes are centrifuged. In both CIAME and temperature-controlled ionic liquid exhaustively dispersive liquid phase microextraction (TILDLME), the dispersion effect can be achieved without a dispersant (Zhou et al., 2008; Bai et al., 2009).

The application of ILs in previously mentioned techniques such as SBME (Guo \& Lee, 2011a) or a three-phase liquid extraction system such as hollow fiber membrane liquid-liquid-liquid microextraction (HFM-LLLME) (Basheer et al., 2008) has also been reported.

Microextraction techniques that use water as an analyte-retaining medium have the greenest characteristics. HF-LPME is one of such techniques. The extraction can be conducted either in a two-phase system, where the aqueous solution of acceptor is separated from the sample by a semipermeable membrane, or in a three-phase system in which the membrane pores are filled with an appropriate organic solvent. In the latter case, the technique is practically solvent-free because the organic solvent is not consumed during the extraction. The isolation of analytes proceeds via appropriate selection of $\mathrm{pH}$ of the acceptor phase and sample (donor). It must be noted that this technique can be applied to ionized analytes only. It is possible to control the selectivity and efficiency of the extraction by appropriately selecting the liquid membrane and the $\mathrm{pH}$ of acceptor and donor phases.

The limited dimensions of the HF-LPME device allowed for the use of electric fields to accelerate the transport of analytes. In this way, an electrochemically-enhanced hollow fiber microextraction technique has been developed (Pedersen-Bjergaard \& Rasmussen, 2006; Jamt et al., 2012). The extraction of analytes takes place in a three-phase system. The analytes migrate via a liquid membrane (immobilized in the pores of a polypropylene fiber) from the aqueous sample, in which the electrode is immersed, to the aqueous acceptor phase inside the fiber where the other electrode 
is present. After the extraction, the acceptor phase is introduced into a CE instrument by means of a syringe. In the case of electrochemically-aided extraction, the transport of analytes is forced by the difference in the potentials of the acceptor and donor phases. Electrochemically-enhanced hollow fiber microextraction enables a significant decrease in extraction time compared to typical HF-LPME. It is noteworthy that a miniaturized version of the technique exists which is analogous to the previously described technique of EME in which the difference in $\mathrm{pH}$ of the acceptor phase and sample was the driving force behind the analyte extraction into the acceptor phase (Ramos Payán et al., 2012). Electrochemically-enhanced extraction allows for very efficient and selective isolation of analytes from samples with complex matrices, including biological and environmental samples (Nojavan \& Fakhari, 2010; Seidi et al., 2011).

Another group of microextraction techniques is distinguished by the use of solid and pseudo-liquid materials in the process of analyte extraction. These techniques constitute the green version of solid phase extraction (SPE) techniques. In the classical version of SPE the analytes are retained on the sorbent bed and then washed out with significant amounts of organic solvents. The additional disadvantage of this type of procedure is the necessary preconcentration of the extract prior to its use in the later steps of the analytical procedure. Preconcentration is usually performed via solvent evaporation. In microextraction techniques, due to the small amount of sorption phase used (even less than $1 \mu \mathrm{L}$ ), the amount of solvents necessary for washing out the analytes is significantly reduced and is a step towards implementing GAC. However, it has to be stressed that the biggest advantages of SPME techniques become apparent when thermal desorption methods are employed to release the analytes. It should be kept in mind that the solvent-free character of a given technique is not directly connected to a concrete technical design because the majority of the presented techniques can also be applied as a variant with solvent extraction. In the two dominant microextraction techniques, i.e., SPME and stir bar sorptive extraction (SBSE) there is a plethora of methodological, technical and material solutions that allow for conducting the analyte isolation with some use of solvents (a step involving washing out of analytes with solvents) as well as completely solvent-free procedures. The analysis of available literature indicates that the above-mentioned division of techniques is mainly based on the type of analytes and sample matrix, and only to the limited extent on miniaturization. However, it is worth mentioning that certain schemes fundamentally result from the fact that small amounts of sorbents are used. The need to achieve low quantification limits forces the use of sorbents which enable obtaining high extraction efficiencies. First of all, this favors the application of adsorbents, and secondly, drives the development of new adsorbent types. On the other hand, the use of adsorbents, which strongly interact with analytes, significantly limits the application of thermal desorption as the method for releasing the analytes. The use of quasiliquid polymers as sorption phase, for example, PDMS, does not mean at all that the desorption of analytes will be exclusively limited to thermal methods. 
Considering the above information, the application of the most popular SPME techniques is discussed in the next part of the chapter, with a particular emphasis placed on the materials used and without the methodology for analyte release.

As already mentioned, SPME is one of the most popular microextraction techniques employing adsorbents. This technique has numerous practical applications which encompass a wide spectrum of analytes and samples in different states of matter and in complex matrices. SPME is one of rare techniques which offers the concurrent fulfillment of a number of requirements posed by GAC, such as complete elimination of organic solvents, simple automation, low labor- and time-intensity procedures for isolating the analytes, and the possibility of in situ and in vivo sample collection (Vuckovic et al., 2010; Zhang et al., 2012). Nevertheless, it should be kept in mind that not all analytical procedures employing this technique fulfill the aforementioned conditions.

Therefore, despite many advantages of SPME, there is still a need to find novel technical, methodological and material solutions which would allow for improving the efficiency of analyte extraction and a further reduction in the sample preparation time. To this end, new designs have been presented, such as internally cooled fibers (Zhang \& Pawliszyn, 1995) and in-tube SPME (Eisert \& Pawliszyn, 1997). Also, information about electrosorption-enhanced solid-phase microextraction (EE-SPME) can be found in the literature (Chai et al., 2007). In the latter technique, a fiber coated with an electrical conductor such as Nafion/carbon nanotubes (Zeng et al., 2010) allows for fiber polarization such that the fiber plays the role of a working electrode. The application of this technique enables a significant decrease in the extraction time and creates a possibility to extract ionizable analytes from samples with polar matrices (Zeng et al., 2011).

However, the greatest progress in SPME techniques has been in the field of novel materials, which aside from increased extraction efficiency should also have better mechanical resistance. These improved features translate into lower material use and higher thermal resistance, which allows for the application of thermal desorption. The most frequently reported sorption materials synthesized for SPME purposes are polymeric sorbents such as conductive polymers (Wu \& Pawliszyn, 2001), molecularly imprinted polymers (MIPs) (Turiel \& Martín-Esteban, 2009), and the materials obtained via sol-gel processes (Kumar et al., 2008). Information about the materials that are more technologically advanced can also be found in the literature, e.g., polymeric ILs whose development has solved the aforementioned problem with low durability of microextraction fibers coated with ILs (Zhao et al., 2008). Polymeric ILs are characterized by high thermal resistance which extend the lifetime of microextraction fibers (up to 150 extractions) (Wanigasekara et al., 2010).

SPME is also suitable for applying nanotechnology products. Nanomaterials are attractive because they possess high surface area and regular and reproducible structure e.g., graphene lattice), while the properties of their adsorption centers can be modified. The system which is most frequently described in literature consists of 
microextraction fibers coated with carbon nanotubes (single-walled carbon nanotubes and MWCNTs). These materials display unusual mechanical resistance and chemical stability as well as unique electrical properties (Wang et al., 2006). Other forms of carbon such as carbon nanocones/disks (Jiménez-Soto et al., 2010), hydroxyfullerenes (Yu et al., 2002), carbon aerogels (CAs) and wormhole-like mesoporous carbons (WMCs) (Zhu et al., 2010) are also used as sorbents. Moreover, graphene has been applied in this context because it has high thermal resistance (above $300^{\circ} \mathrm{C}$ ) (J. Chen et al., 2010). Meso- and nanoporous silica materials have even higher thermal resistance reaching $900{ }^{\circ} \mathrm{C}$, for example, commercially available MCM-41 and SBA-15 (Du et al., 2005). In the literature, there are also reports about silica-based composite materials such as nanocomposite polypyrrole and SBA-15 (Gholivand et al., 2011), silica-carbon composites (Zeng et al., 2008) and the mechanically-resistant composite of aniline and silica nanoparticles (Bagheri \& Roostaie, 2012). An interesting solution is the techniques in which a metal wire is coated with silica microstructures called flower-like. The created highly porous coating serves as a support for liquid organic extractant (typically propyl benzoate). After extraction, both the extractant and retained analytes are evaporated in the GC injection port due to their high volatility. Coatings of gold and silver nanoparticles are other nanosorbents described in the literature. The obtained adsorption layer is characterized by very high thermal and chemical resistance (Feng et al., 2012).

The second-most reported microextraction technique which fulfills the principles of GAC is SBSE. It was described for the first time in 1999 as an alternative to SPME and has a similar range of applications. In principle, SBSE was developed to fulfill the need for large sorbent volumes (compared to SPME) and to retain the simplicity of operation. This technique allows for partial or even total elimination of organic solvents from the analytical procedure, which has been achieved by the application of sorbents that are identical or similar to the ones used in SPME (Baltussen et al., 1997). Extraction can be conducted directly from the sample or from the sample headspace. The analytes can be released via thermal desorption as well as desorption with a solvent (Kawaguchi et al., 2006). As in the case of SPME, significant progress in the field of sorbent development has been made in SBSE techniques although its scope is more limited since SBSE requires high mechanical resistance (e.g., to friction on the walls of the sample container). Nevertheless, besides the typical sorbents such as PDMS, a number of new materials can be used such as polyurethane foams (PU) (Portugal et al., 2008; Silva et al., 2008), poly(phthalazine ether sulfone ketone) (PPESK) (Guan et al., 2008), alkyl-diol-silica (ADS) restricted access material (RAM) (Lambert et al., 2005), monolithic adsorbents (Huang et al., 2009a, 2009b, 2009c), and composite sorbents such as PDMS/polypyrrole (PPY) (Melo et al., 2009). The descriptions of coating a stirrer with MIP-type materials have also been published (Zhu et al., 2006).

Aside from the two leading techniques described above, many novel designs can be found in the literature. This indicates that a continuous need for technical 
solutions still exists in order to decrease the amount of solvents that are used. For example, in micro-SPE ( $\mu$-SPE) a solid sorbent is placed inside a bag made of a porous membrane (Basheer et al., 2006). The use of the membrane allows one to extract the analyte from contaminated samples and suspensions. Moreover, in comparison to classical SPE, $\mu$-SPE is simpler and it decreases the time needed for analyte extraction (Basheer et al., 2007). A comparatively simple way of introducing sorbent into the sample was presented in adsorptive microextraction techniques, such as gluing the sorbent to a carrier made of polypropylene fibers or polystyrene spheres (Neng et al., 2010). Stir cake sorptive extraction (SCSE) is an interesting solution which extends the life span of sorptive elements because the monolithic adsorbent has been synthesized inside the holder that protects its content against mechanical damage. In this way, the life span of a sorptive element can reach up to $1000 \mathrm{~h}$ of extraction (Huang et al., 2011). Solid phase nanoextraction (SPNE) techniques are also worth mentioning because they are similar, to a certain degree, to dispersive techniques although in this case a solution of gold nanoparticles is used as suspension (Wang \& Campiglia, 2008).

Unfortunately, in the techniques just described it is necessary to desorb the analytes with solvents, which is a disadvantage. However, it has to be highlighted that the use of solvents has been significantly reduced due to the very small dimensions of the extraction devices.

\subsection{Automation in Microextraction Techniques}

Possible automation of sample preparation is a significant aspect used to evaluate whether a procedure fulfills the principles of GAC. When making such an evaluation, it is critical to consider both the possible automation of sample preparation and the possible automation of the entire analytical procedure, including the introduction of analytes into a measuring instrument. Based on the analysis of potential cost reduction of materials and energy used, it seems that a possible automation of the entire analytical procedure has much lower impact on the overall costs. On the other hand, the automation of sample preparation translates into a direct decrease of materials used, including organic solvents when applicable. This mainly results from the reduction of human error, which increases the reproducibility of the results and therefore reduces the number of necessary analyses. Another obvious consequence of automation is the reduced generation of waste, lower energy use for obtaining a sufficient amount of data, and lower costs of data analysis. Considering the miniaturization of microextraction devices, it seems that the automation of microextraction techniques should not pose big problems. However, analysis of published literature shows that miniaturization may help with automation, but it is not always the case. In some techniques, e.g., SPME, the automation happened almost naturally because it has been based on the previously automated devices. In the case of other techniques, it was 
necessary to develop specialized technological solutions. The examples of different technical solutions used in the aforementioned microextraction techniques are presented below.

The automation of SPME (not always implemented) has been achieved by designing an appropriate holder for the SPME device which is compatible with typical autosamplers. In this way, it is possible to conduct all steps of the SPME procedure in fully automated mode, i.e., sample incubation, analyte extraction, desorption and fiber cleaning. Moreover, thanks to the application of computer-controlled integrated systems, the critical parameters such as extraction time and sample temperature can be controlled (O'Reilly et al., 2005; Vuckovic et al., 2008). At present, a number of commercial devices are available, including TriPlus (Thermo Fisher Scientific; Milan, Italy), Combi-PAL (CTC Analytics; Zwingen, Switzerland), MPS 2(Gerstel Inc.; Mulheim and der Ruhr, Germany), and Concept 96 (PAS Technology; Magdala, Germany). Modifications allowing for multiple concurrent extractions are also known, for example, multi-well plate format SPME. These have helped to reduce the time necessary for obtaining the final quantification, and in the improvement of technique precision and extraction repeatability (Vatinno et al., 2008). Some variants of SPME have also been automated, like in the case of internally-cooled coated fiber (CCF) device. Here the automation enables a better control of fiber temperature as well as the application of the CCF-SPME device in routine analyses (Chen \& Pawliszyn, 2006).

Procedure automation is also possible in the case of SBSE techniques. Moreover, devices for analyte desorption have been introduced to analytical practices which allow for concurrent determination of analytes released from a number of sorption elements (Kawaguchi et al., 2004).

In general, the attempts to automate liquid phase extraction techniques have encountered bigger difficulties. Nevertheless, examples of successful automation of the sample preparation step exist.

The development of an automated version of SDME was relatively simple. The variant is based on a typical microsyringe which is coupled with an automatic sample feeder (Ouyang et al., 2007). A successful attempt at automating the analytical procedure was also undertaken in the case of HF-LPME. In addition, in the dynamic hollow-fiber liquid-phase microextraction (dynamic-HF-LPME) the possibility of concurrent analyte sampling from a number of samples has been achieved (Pezo et al., 2007). Information about the fully automated procedures based on DLLME techniques can also be found in the published literature. SI-DLLME enables on-line dispensing of the extraction phase samples into FAAS (Anthemidis \& Ioannou, 2009) and ETAAS atomizers. In this technique, a dispersing agent, extractant and complexing compound is introduced into the stream of aqueous sample which flows through a microcolumn filled with adsorbent. Next, the retained complexes of analytes are washed out with isobutyl methyl ketone directly into the atomizer of an atomic absorption spectrometer (Anthemidis \& Ioannou, 2011). In another, more complicated technical solution, the extraction is 
conducted in a conical vial, followed by spontaneous phase separation. The extract is transferred into a micro-volume Z-flow cell and then analyzed spectrophotometrically (Andruch et al., 2012).

\subsection{Chemometric Approaches for Optimization and Evaluation of Microextraction Techniques}

Basically, all techniques of analyte isolation apply a similar scheme, i.e., contact between the sample and the sorption phase, the latter displaying higher affinity for the analytes than the sample matrix. As a result, the analytes are collected on the sorbent in accordance with the system-specific partition coefficient and the ratio of sorption phase volume to sample volume. The kinetics of analyte transport depend on the values of the diffusion coefficient and the geometry of the system. The extraction efficiency in microextraction techniques is fundamentally disadvantageous because of the volume ratio of phases. As a consequence, exhaustive extraction occurs only in very rare cases. In most situations, the amount of analyte retained during the extraction is sufficient to deplete their content in the sample to the level which can be assumed to be constant. Under these circumstances, the precise determination of optimal extraction conditions has a fundamental role. In-depth knowledge of physicochemical processes taking place in the system during extraction is necessary for selecting critical parameters which should be considered as independent variables in the optimization process. Nevertheless, the quantitative effect of these parameters, particularly in the case of interactions among variables, is difficult to predict. In such situations, the classical methodology for establishing optimal conditions of analyte isolation fails completely because it is based on the one-factor-attime approach in which only one parameter is optimized and the others are treated as constant. At this point, the application of chemometrics becomes necessary because it offers more advanced tools based on mathematical and statistical methods. In the optimally designed set of experiments, chemometrics can fulfill the need for finding the maximum amount of information which will allow for determining optimal conditions of chemical processes and extending the knowledge about the system operation. The aforementioned aspects of chemometric applications implement the principles of GAC in several ways:

1. highlighting the parameters which are significant for the process of analyte extraction and therefore creating possibilities to reduce the number of variables being optimized, and gaining the full control over the sample preparation process

2. a significant reduction in the number of experiments that are necessary to determine the optimal conditions of analyte isolation

3. a possibility to determine optimal conditions irrespective of interactions, which guarantees the most effective procedure for analyte isolation. 


\section{STEPS IN CHEMOMETRIC OPTIMIZATION OF MICROEXTRACTION}

I. Definition of the problem and selection of the appropriate variables and responses through screening studies.

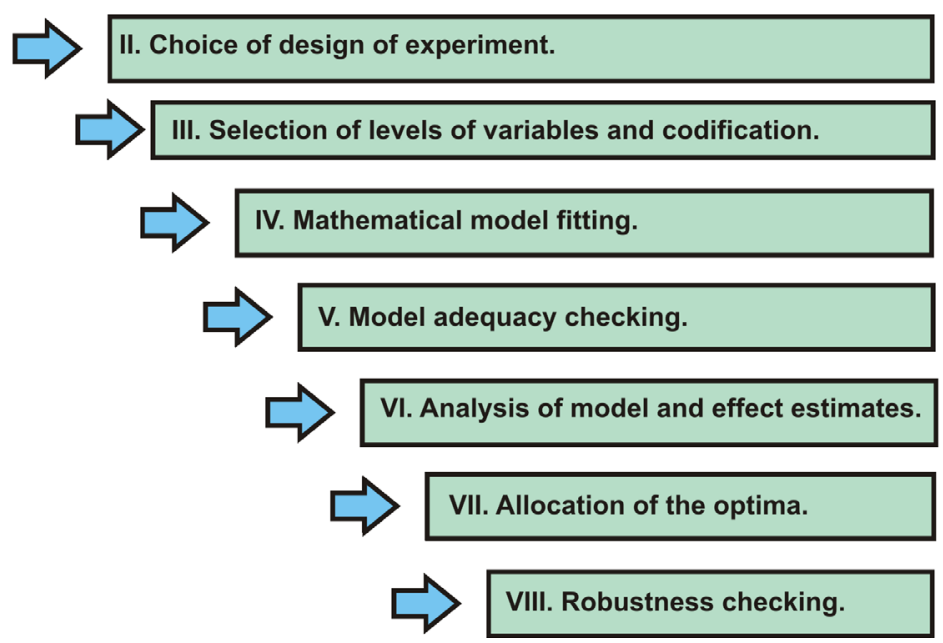

Figure 8.3 Steps in chemometric optimization of microextraction.

As mentioned before, a key issue from the point of view of microextraction techniques is the inclusion of interactions among the variables. To this end, an appropriate mathematical model (equation 8.1) is used, which is fitted to the input data describing the process parameters $\left(x_{i}, x_{j}\right)$ and the system response $(Y)$, the latter usually being the parameter related to the amount of analytes retained (e.g., the area of a chromatographic peak).

$Y=\beta_{0}+\sum \beta_{i} x_{i}+\sum \beta_{i i} x_{i}^{2}+\sum \beta_{i j} x_{i} x_{j}+\varepsilon$

where $\beta_{0}$ is constant, $\beta_{i}$ is a regression coefficient of linear terms, $\beta_{i i}$ is a regression coefficient of quadratic terms, $\beta_{i j}$ is a regression coefficient of interactions between the parameters $x_{i}$ and $x_{j}$, and $\varepsilon$ is the residual associated with the experiment.

Equation 8.1 is presented in the most generalized form which allows for determining the linear effects, maximal values or saddle points, and the interactions between the parameters. In practice, the number of terms in equation 8.1 is determined from the goodness of fit analysis (analysis of variance, ANOVA) using the observed values. The optimization of microextraction process (with respect to the number of variables, Figure 8.3) is conducted in a number of key steps. Most of the steps are realized by utilizing chemometric tools, while the experimenter has the fundamental influence 
on the final results with regard to the appropriate choice of optimized parameters (step 1) and the determination of their variation level (step 3).

As mentioned before, a number of parameters should be considered when dealing with microextraction techniques. These range from the most general ones, such as temperature, sample volume (or headspace volume) and extraction time, to more specific parameters, including extractant type and volume, extraction mode (direct or headspace), sample $\mathrm{pH}$, agitation type (including the stirring rate) and ionic strength. In addition, technique-specific parameters such as the volume of dispersant and emulsifying agents or the electrical potential must be considered and optimized. Table 8.1 contains examples of literature data about the optimization of the sample preparation process using some microextraction techniques, as well as the applied models. A detailed description of specific models goes beyond the scope of this chapter so the reader wishing to broaden his/her knowledge in this field is referred to the specialized literature.

\subsection{Conclusions}

The miniaturization of sample preparation techniques fits directly into the trend set by the principles of GAC. The resulting reduction in the amount of organic solvents used during the isolation of analytes and their subsequent release is practically unquestionable. Miniaturization also produces less waste than in conventional systems. Nevertheless, the evaluation based exclusively on this one indicator leads to superficial assessment which does not reflect the whole picture. Therefore, we would like to turn the reader's attention to a couple of additional aspects of microextraction techniques which should be developed further in order to implement the majority of GAC principles. Doubts arise when we consider not only the final application of some materials, but also the process of their production. MIPs and ILs can serve as an example here. In both cases, the amount of chemical reagents used in the synthesis and refining of these compounds (ILs in particular) can outweigh the amount of reagents saved due to the application of new materials in the isolation of analytes. The possibility of automating the sample preparation technique and coupling it directly to the final measurement step is another important factor. The operator-independent procedures have better reproducibility of results and throughput; therefore, they are characterized by a significantly lower use of materials and energy per analytical process. In this context, the shortcomings are noticeable, particularly in the liquid phase extraction techniques. In this case, the sample preparation step is quite often automated while the introduction of the sample into a measuring instrument is, with rare exceptions, a manual process. It seems that despite huge creativity of analytical chemists with respect to novel technical solutions, during the designing process more attention still should be paid to the possibility of future automation of both the sample preparation step alone and the entire analytical procedure. Also, the analysis of published litera- 


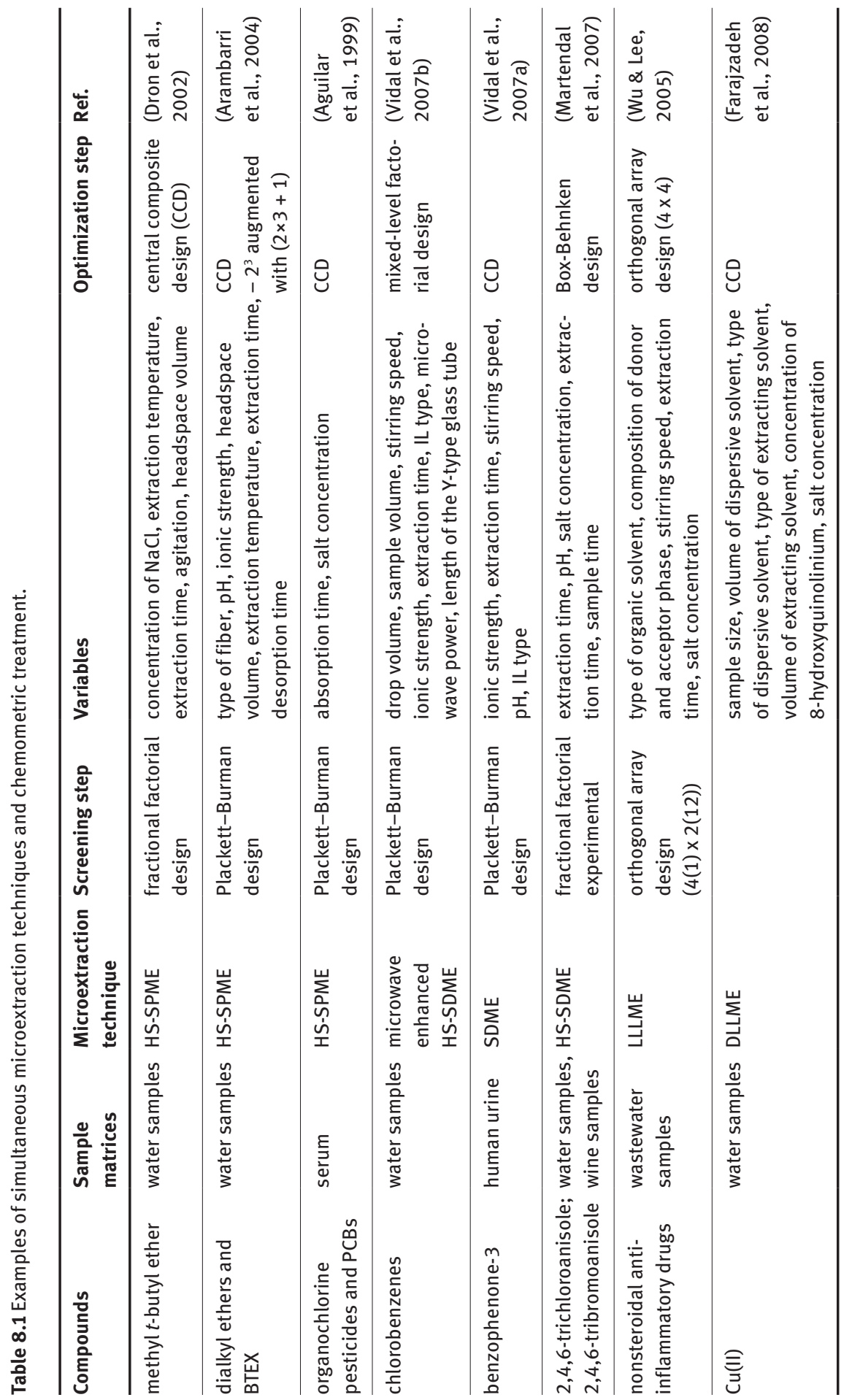


ture indicates that the potential presented by chemometric tools is underutilized. At present, the one-factor-at-a-time method of optimization still dominates, while this approach should be used only for the simplest systems, particularly with regard to microextraction techniques.

Obviously, the aforementioned remarks do not discredit the enormous impact of miniaturization as one of many ways of fulfilling the requirements posed by GAC. They rather point to the fact that the full implementation of GAC principles demands additional activities with respect to miniaturization in order to fully live up to the GAC designation.

\section{Abbreviations}

\begin{tabular}{|c|c|}
\hline$\mu-\mathrm{SPE}$ & micro-solid phase extraction \\
\hline ADS & alkyl-diol-silica \\
\hline ANOVA & analysis of variance \\
\hline $\mathrm{A} \mu \mathrm{E}$ & adsorptive $\mu$-extraction \\
\hline CAs & carbon aerogels \\
\hline CCD & Central Composite Design \\
\hline CE & capillary electrophoresis \\
\hline CIAME & cold-induced aggregation microextraction \\
\hline CPE & cloud point extraction \\
\hline $\mathrm{dCPE}$ & dual cloud point extraction \\
\hline DD-SDME & drop-to-drop single-drop microextraction \\
\hline DLLME & dispersive liquid-liquid microextraction \\
\hline DLLME-SFO & $\begin{array}{l}\text { dispersive liquid-liquid microextraction based on the solidifica- } \\
\text { tion of a floating organic drop }\end{array}$ \\
\hline dLPME & dynamic liquid-phase microextraction \\
\hline DSDME & directly suspended droplet microextraction technique \\
\hline dynamic-HF-LPME & dynamic hollow-fiber liquid-phase microextraction \\
\hline EE-SPME & electrosorption enhanced solid-phase microextraction \\
\hline EME & electro membrane extraction \\
\hline ETAAS & electrothermal atomic absorption spectrometry \\
\hline FAAS & flame atomic absorption spectrometry \\
\hline GC & gas chromatography \\
\hline HF-LPME & hollow fiber liquid phase microextraction \\
\hline HF-SLPME & Hollow fiber solid-liquid phase microextraction \\
\hline HPLC & high performance liquid chromatography \\
\hline HS-SDME & headspace single-drop microextraction \\
\hline IL & ionic liquid \\
\hline IP-SAME & ion pair based surfactant assisted microextraction \\
\hline ISFME & in situ solvent-formation microextraction \\
\hline
\end{tabular}




\begin{tabular}{|c|c|}
\hline LLE & liquid-liquid extraction \\
\hline LPME & liquid-phase microextraction \\
\hline MIPs & molecularly imprinted polymers \\
\hline MWCNT & multiwall carbon nanotubes \\
\hline NPOE & 2-nitrophenyl octyl ether \\
\hline PAHs & polycyclic aromatic hydrocarbons \\
\hline PDMS & polydimethylsiloxane \\
\hline PMMA & $\operatorname{poly}($ methyl)methacrylate \\
\hline PPESK & poly(phthalazine ether sulfone ketone) \\
\hline PPY & polypyrrole \\
\hline PU & polyurethane foam \\
\hline RAM & restricted access material \\
\hline SA-DLLME & surfactant-assisted dispersive liquid-liquid microextraction \\
\hline SBME & solvent bar microextraction \\
\hline SBSE & stir bar sorptive extraction \\
\hline SC-DHF-HS-LPME & $\begin{array}{l}\text { solvent cooling assisted dynamic hollow-fiber-supported head- } \\
\text { space liquid phase microextraction }\end{array}$ \\
\hline SCSE & stir cake sorptive extraction \\
\hline SD-DLLME & solvent demulsification dispersive liquid-liquid microextraction \\
\hline SDME & single-drop microextraction \\
\hline SFODME & Solidified floating organic drop microextraction \\
\hline SI-DLLME & sequential injection dispersive liquid-liquid microextraction \\
\hline SM-DLLME & supramolecular-based dispersive liquid-liquid microextraction \\
\hline SPE & solid phase extraction \\
\hline SPME & solid-phase microextraction \\
\hline SPNE & solid phase nanoextraction \\
\hline ST-DLLME & solvent terminated dispersive liquid-liquid microextraction \\
\hline TILDLME & $\begin{array}{l}\text { temperature-controlled ionic liquid exhaustively dispersive liquid } \\
\text { phase micro-extraction }\end{array}$ \\
\hline UASEME & $\begin{array}{l}\text { ultrasound-assisted surfactant-enhanced emulsification microex- } \\
\text { traction }\end{array}$ \\
\hline USA-DLLME & ultrasound-assisted dispersive liquid-liquid microextraction \\
\hline USAEME & ultrasound-assisted emulsification-microextraction \\
\hline US-DLLME & ultrasound-assisted dispersive liquid-liquid microextraction \\
\hline VALLME & vortex-assisted liquid-liquid microextraction \\
\hline VSLLME & $\begin{array}{l}\text { vortex-assisted surfactant-enhanced-emulsification liquid-liquid } \\
\text { microextraction }\end{array}$ \\
\hline
\end{tabular}




\section{References}

Aguilar, C., Peñalver, A., Pocurull, E., Ferré, J., Borrull, F., \& Marcé, R. M. (1999). Optimization of solid-phase microextraction conditions using a response surface methodology to determine organochlorine pesticides in water by gas chromatography and electron-capture detection. Journal of Chromatography A, 844, 425-432.

Aguilera-Herrador, E., Lucena, R., Cárdenas, S., \& Valcárcel, M. (2007). Direct coupling of ionic liquid based single-drop microextraction and GC/MS. Analytical Chemistry, 80, 793-800.

Aguilera-Herrador, E., Lucena, R., Cárdenas, S., \& Valcárcel, M. (2008). Ionic liquid-based single-drop microextraction/gas chromatographic/mass spectrometric determination of benzene, toluene, ethylbenzene and xylene isomers in waters. Journal of Chromatography $A$, 1201, 106-111.

Andruch, V., Acebal, C. C., Škrlíková, J., Sklenářová, H., Solich, P., Balogh, I. S., et al. (2012). Automated on-line dispersive liquid-liquid microextraction based on a sequential injection system. Microchemical Journal, 100, 77-82.

Anthemidis, A. N., \& Ioannou, K.-I. G. (2009). On-line sequential injection dispersive liquid-liquid microextraction system for flame atomic absorption spectrometric determination of copper and lead in water samples. Talanta, 79, 86-91.

Anthemidis, A. N., \& loannou, K.-I. G. (2010). Development of a sequential injection dispersive liquid-liquid microextraction system for electrothermal atomic absorption spectrometry by using a hydrophobic sorbent material: Determination of lead and cadmium in natural waters. Analytica Chimica Acta, 668, 35-40.

Anthemidis, A. N., \& loannou, K.-I. G. (2011). Sequential injection dispersive liquid-liquid microextraction based on fatty alcohols and poly(etheretherketone)-turnings for metal determination by flame atomic absorption spectrometry. Talanta, 84, 1215-1220.

Arambarri, I., Lasa, M., Garcia, R., \& Millán, E. (2004). Determination of fuel dialkyl ethers and BTEX in water using headspace solid-phase microextraction and gas chromatography-flame ionization detection. Journal of Chromatography A, 1033, 193-203.

Arvand, M., Bozorgzadeh, E., Shariati, S., \& Zanjanchi, M. A. (2012). Trace determination of linear alkylbenzene sulfonates using ionic liquid based ultrasound-assisted dispersive liquid-liquid microextraction and response surface methodology. Analytical Methods, 4, 2272-2277.

Baghdadi, M., \& Shemirani, F. (2008). Cold-induced aggregation microextraction: A novel sample preparation technique based on ionic liquids. Analytica Chimica Acta, 613, 56-63.

Bagheri, H., \& Roostaie, A. (2012). Aniline-silica nanocomposite as a novel solid phase microextraction fiber coating. Journal of Chromatography A, 1238, 22-29.

Bai, H., Zhou, Q., Xie, G., \& Xiao, J. (2009). Enrichment and sensitive determination of dichlorodiphenyltrichloroethane and its metabolites with temperature controlled ionic liquid dispersive liquid phase microextraction prior to high performance liquid phase chromatography. Analytica Chimica Acta, 651, 64-68.

Baltussen, E., Janssen, H.-G., Sandra, P., \& Cramers, C. A. (1997). A new method for sorptive enrichment of gaseous samples: Application in air analysis and natural gas characterization. Journal of High Resolution Chromatography, 20, 385-393.

Basheer, C., Alnedhary, A. A., Madhava Rao, B. S., Balasubramanian, R., \& Lee, H. K. (2008). Ionic liquid supported three-phase liquid-liquid-liquid microextraction as a sample preparation technique for aliphatic and aromatic hydrocarbons prior to gas chromatography-mass spectrometry. Journal of Chromatography A, 1210, 19-24.

Basheer, C., Alnedhary, A. A., Rao, B. S. M., Valliyaveettil, S., \& Lee, H. K. (2006). Development and application of porous membrane-protected carbon nanotube micro-solid-phase extraction combined with gas chromatography/mass spectrometry. Analytical Chemistry, 78, 2853-2858. 
Basheer, C., Chong, H. G., Hii, T. M., \& Lee, H. K. (2007). Application of porous membraneprotected micro-solid-phase extraction combined with HPLC for the analysis of acidic drugs in wastewater. Analytical Chemistry, 79, 6845-6850.

Batlle, R., López, P., Nerín, C., \& Crescenzi, C. (2008). Active single-drop microextraction for the determination of gaseous diisocyanates. Journal of Chromatography A, 1185, 155-160.

Chai, X., He, Y., Ying, D., Jia, J., \& Sun, T. (2007). Electrosorption-enhanced solid-phase microextraction using activated carbon fiber for determination of aniline in water. Journal of Chromatography A, 1165, 26-31.

Chamsaz, M., Atarodi, A., Eftekhari, M., Asadpour, S., \& Adibi, M. (2013). Vortex-assisted ionic liquid microextraction coupled to flame atomic absorption spectrometry for determination of trace levels of cadmium in real samples. Journal of Advanced Research, 4, 35-41.

Chen, H., Chen, R., \& Li, S. (2010). Low-density extraction solvent-based solvent terminated dispersive liquid-liquid microextraction combined with gas chromatography-tandem mass spectrometry for the determination of carbamate pesticides in water samples. Journal of Chromatography A, 1217, 1244-1248.

Chen, J., Zou, J., Zeng, J., Song, X., Ji, J., Wang, Y., et al. (2010). Preparation and evaluation of graphene-coated solid-phase microextraction fiber. Analytica Chimica Acta, 678, 44-49.

Chen, Y., \& Pawliszyn, J. (2006). Miniaturization and automation of an internally cooled coated fiber device. Analytical Chemistry, 78, 5222-5226.

Chisvert, A., Román, I. P., Vidal, L., \& Canals, A. (2009). Simple and commercial readily-available approach for the direct use of ionic liquid-based single-drop microextraction prior to gas chromatography: Determination of chlorobenzenes in real water samples as model analytical application. Journal of Chromatography A, 1216, 1290-1295.

Cruz-Vera, M., Lucena, R., Cárdenas, S., \& Valcárcel, M. (2008). Ionic liquid-based dynamic liquid-phase microextraction: Application to the determination of anti-inflammatory drugs in urine samples. Journal of Chromatography A, 1202, 1-7.

Dron, J., Garcia, R., \& Millán, E. (2002). Optimization of headspace solid-phase microextraction by means of an experimental design for the determination of methyl tert.-butyl ether in water by gas chromatography-flame ionization detection. Journal of Chromatography A, 963, 259-264.

Du, X.-Z., Wang, Y.-R., Tao, X.-J., \& Deng, H.-L. (2005). An approach to application of mesoporous hybrid as a fiber coating of solid-phase microextraction. Analytica Chimica Acta, 543, 9-16.

Eisert, R., \& Pawliszyn, J. (1997). New trends in solid phase microextraction. Critical Reviews in Analytical Chemistry, 27, 103-135.

Es'haghi, Z., Golsefidi, M. A., Saify, A., Tanha, A. A., Rezaeifar, Z., \& Alian-Nezhadi, Z. (2010). Carbon nanotube reinforced hollow fiber solid/liquid phase microextraction: A novel extraction technique for the measurement of caffeic acid in Echinacea purpurea herbal extracts combined with high-performance liquid chromatography. Journal of Chromatography A, 1217, 2768-2775.

Es'haghi, Z., Khooni, M. A.-K., \& Heidari, T. (2011). Determination of brilliant green from fish pond water using carbon nanotube assisted pseudo-stir bar solid/liquid microextraction combined with UV-vis spectroscopy-diode array detection. Spectrochimica Acta Part A: Molecular and Biomolecular Spectroscopy, 79, 603-607.

Farajzadeh, M. A., Bahram, M., Mehr, B. G., \& Jönsson, J. Å. (2008). Optimization of dispersive liquid-liquid microextraction of copper (II) by atomic absorption spectrometry as its oxinate chelate: Application to determination of copper in different water samples. Talanta, 75, 832-840.

Farajzadeh, M. A., Djozan, D., \& Khorram, P. (2012). Development of a new dispersive liquid-liquid microextraction method in a narrow-bore tube for preconcentration of triazole pesticides from aqueous samples. Analytica Chimica Acta, 713, 70-78. 
Farajzadeh, M. A., Seyedi, S. E., Shalamzari, M. S., \& Bamorowat, M. (2009). Dispersive liquid-liquid microextraction using extraction solvent lighter than water. Journal of Separation Science, 32, 3191-3200.

Feng, J., Sun, M., Li, J., Liu, X., \& Jiang, S. (2011). A novel silver-coated solid-phase microextraction metal fiber based on electroless plating technique. Analytica Chimica Acta, 701, 174-180.

Feng, J., Sun, M., Li, J., Liu, X., \& Jiang, S. (2012). A novel aromatically functional polymeric ionic liquid as sorbent material for solid-phase microextraction. Journal of Chromatography A, 1227, 54-59.

Fontana, A. R., Wuilloud, R. G., Martínez, L. D., \& Altamirano, J. C. (2009). Simple approach based on ultrasound-assisted emulsification-microextraction for determination of polibrominated flame retardants in water samples by gas chromatography-mass spectrometry. Journal of Chromatography A, 1216, 147-153.

Gholivand, M. B., Abolghasemi, M. M., \& Fattahpour, P. (2011). Polypyrrole/hexagonally ordered silica nanocomposite as a novel fiber coating for solid-phase microextraction. Analytica Chimica Acta, 704, 174-179.

Giokas, D., Paleologos, E., \& Karayannis, M. (2002). Speciation of Fe(II) and Fe(III) by the modified ferrozine method, FIA-spectrophotometry, and flame AAS after cloud-point extraction. Analytical and Bioanalytical Chemistry, 373, 237-243.

Guan, W., Wang, Y., Xu, F., \& Guan, Y. (2008). Poly(phthalazine ether sulfone ketone) as novel stationary phase for stir bar sorptive extraction of organochlorine compounds and organophosphorus pesticides. Journal of Chromatography A, 1177, 28-35.

Guo, L., \& Lee, H. K. (2011a). Ionic liquid based three-phase liquid-liquid-liquid solvent bar microextraction for the determination of phenols in seawater samples. Journal of Chromatography A, 1218, 4299-4306.

Guo, L., \& Lee, H. K. (2011b). Low-density solvent-based solvent demulsification dispersive liquidliquid microextraction for the fast determination of trace levels of sixteen priority polycyclic aromatic hydrocarbons in environmental water samples. Journal of Chromatography $A, 1218$, 5040-5046.

Hashemi, P., Beyranvand, S., Mansur, R. S., \& Ghiasvand, A. R. (2009). Development of a simple device for dispersive liquid-liquid microextraction with lighter than water organic solvents: Isolation and enrichment of glycyrrhizic acid from licorice. Analytica Chimica Acta, 655, 60-65.

He, Y., Pohl, J., Engel, R., Rothman, L., \& Thomas, M. (2009). Preparation of ionic liquid based solid-phase microextraction fiber and its application to forensic determination of methamphetamine and amphetamine in human urine. Journal of Chromatography A, 1216, 4824-4830.

Ho, T. D., Canestraro, A. J., \& Anderson, J. L. (2011). Ionic liquids in solid-phase microextraction: A review. Analytica Chimica Acta, 695, 18-43.

Hsieh, Y.-N., Huang, P.-C., Sun, I. W., Whang, T.-J., Hsu, C.-Y., Huang, H.-H., \& Kuei, C.-H. (2006). Nafion membrane-supported ionic liquid-solid phase microextraction for analyzing ultra trace PAHs in water samples. Analytica Chimica Acta, 557, 321-328.

Huang, S.-P., \& Huang, S.-D. (2007). Determination of organochlorine pesticides in water using solvent cooling assisted dynamic hollow-fiber-supported headspace liquid-phase microextraction. Journal of Chromatography $A, 1176,19-25$.

Huang, X., Chen, L., Lin, F., \& Yuan, D. (2011). Novel extraction approach for liquid samples: Stir cake sorptive extraction using monolith. Journal of Separation Science, 34, 2145-2151.

Huang, X., Lin, J., Yuan, D., \& Hu, R. (2009a). Determination of steroid sex hormones in wastewater by stir bar sorptive extraction based on poly(vinylpyridine-ethylene dimethacrylate) monolithic material and liquid chromatographic analysis. Journal of Chromatography A, 1216, 3508-3511. 
Huang, X., Qiu, N., Yuan, D., \& Huang, B. (2009b). A novel stir bar sorptive extraction coating based on monolithic material for apolar, polar organic compounds and heavy metal ions. Talanta, 78, 101-106.

Huang, X., Qiu, N., Yuan, D., \& Lin, Q. (2009c). Sensitive determination of strongly polar aromatic amines in water samples by stir bar sorptive extraction based on poly(vinylimidazole-divinylbenzene) monolithic material and liquid chromatographic analysis. Journal of Chromatography A, 1216, 4354-4360.

Hutchinson, J. P., Setkova, L., \& Pawliszyn, J. (2007). Automation of solid-phase microextraction on a 96-well plate format. Journal of Chromatography A, 1149, 127-137.

Jafarvand, S., \& Shemirani, F. (2011). Supramolecular-based dispersive liquid-liquid microextraction: A novel sample preparation technique utilizes coacervates and reverse micelles. Journal of Separation Science, 34, 455-461.

Jamt, R. E. G., Gjelstad, A., Eibak, L. E. E., Øiestad, E. L., Christophersen, A. S., Rasmussen, K. E., \& Pedersen-Bjergaard, S. (2012). Electromembrane extraction of stimulating drugs from undiluted whole blood. Journal of Chromatography A, 1232, 27-36.

Jeannot, M. A., \& Cantwell, F. F. (1996). Solvent microextraction into a single drop. Analytical Chemistry, 68, 2236-2240.

Jia, C., Zhu, X., Wang, J., Zhao, E., He, M., Chen, L., \& Yu, P. (2010). Extraction of pesticides in water samples using vortex-assisted liquid-liquid microextraction. Journal of Chromatography $A$, $1217,5868-5871$.

Jiang, X., \& Lee, H. K. (2004). Solvent bar microextraction. Analytical Chemistry, 76, 5591-5596.

Jiménez-Soto, J. M., Cárdenas, S., \& Valcárcel, M. (2010). Carbon nanocones/disks as new coating for solid-phase microextraction. Journal of Chromatography A, 1217, 3341-3347.

Kawaguchi, M., Ishii, Y., Sakui, N., Okanouchi, N., Ito, R., Inoue, K., et al. (2004). Stir bar sorptive extraction with in situ derivatization and thermal desorption-gas chromatography-mass spectrometry in the multi-shot mode for determination of estrogens in river water samples. Journal of Chromatography A, 1049, 1-8.

Kawaguchi, M., Ito, R., Saito, K., \& Nakazawa, H. (2006). Novel stir bar sorptive extraction methods for environmental and biomedical analysis. Journal of Pharmaceutical and Biomedical Analysis, 40, 500-508.

Khalili Zanjani, M. R., Yamini, Y., Shariati, S., \& Jönsson, J. Å. (2007). A new liquid-phase microextraction method based on solidification of floating organic drop. Analytica Chimica Acta, 585, 286-293.

Kosaraju, P. B., \& Sirkar, K. K. (2007). Novel solvent-resistant hydrophilic hollow fiber membranes for efficient membrane solvent back extraction. Journal of Membrane Science, 288, 41-50.

Kumar, A., Gaurav, Malik, A. K., Tewary, D. K., \& Singh, B. (2008). A review on development of solid phase microextraction fibers by sol-gel methods and their applications. Analytica Chimica Acta, 610,1-14.

Lambert, J.-P., Mullett, W. M., Kwong, E., \& Lubda, D. (2005). Stir bar sorptive extraction based on restricted access material for the direct extraction of caffeine and metabolites in biological fluids. Journal of Chromatography A, 1075, 43-49.

Leong, M.-I., \& Huang, S.-D. (2008). Dispersive liquid-liquid microextraction method based on solidification of floating organic drop combined with gas chromatography with electron-capture or mass spectrometry detection. Journal of Chromatography A, 1211, 8-12.

Liu, H., \& Dasgupta, P. K. (1996). Analytical chemistry in a drop. Solvent extraction in a microdrop. Analytical Chemistry, 68, 1817-1821.

Liu, J.-f., Jiang, G.-b., Chi, Y.-g., Cai, Y.-q., Zhou, Q.-x., \& Hu, J.-T. (2003). Use of ionic liquids for liquid-phase microextraction of polycyclic aromatic hydrocarbons. Analytical Chemistry, 75, 5870-5876. 
Liu, J.-f., Li, N., Jiang, G.-b., Liu, J.-m., Jönsson, J. Å., \& Wen, M.-j. (2005). Disposable ionic liquid coating for headspace solid-phase microextraction of benzene, toluene, ethylbenzene, and xylenes in paints followed by gas chromatography-flame ionization detection. Journal of Chromatography A, 1066, 27-32.

López-Darias, J., Pino, V., Anderson, J. L., Graham, C. M., \& Afonso, A. M. (2010). Determination of water pollutants by direct-immersion solid-phase microextraction using polymeric ionic liquid coatings. Journal of Chromatography A, 1217, 1236-1243.

Madej, K. (2009). Microwave-assisted and cloud-point extraction in determination of drugs and other bioactive compounds. TrAC Trends in Analytical Chemistry, 28, 436-446.

Martendal, E., Budziak, D., \& Carasek, E. (2007). Application of fractional factorial experimental and Box-Behnken designs for optimization of single-drop microextraction of 2,4,6-trichloroanisole and 2,4,6-tribromoanisole from wine samples. Journal of Chromatography A, 1148, 131-136.

Martinis, E. M., Olsina, R. A., Altamirano, J. C., \& Wuilloud, R. G. (2008). Sensitive determination of cadmium in water samples by room temperature ionic liquid-based preconcentration and electrothermal atomic absorption spectrometry. Analytica Chimica Acta, 628, 41-48.

Melo, L. P., Nogueira, A. M., Lanças, F. M., \& Queiroz, M. E. C. (2009). Polydimethylsiloxane/ polypyrrole stir bar sorptive extraction and liquid chromatography (SBSE/LC-UV) analysis of antidepressants in plasma samples. Analytica Chimica Acta, 633, 57-64.

Moradi, M., Yamini, Y., Kakehmam, J., Esrafili, A., \& Ghambarian, M. (2011). A new strategy to simultaneous microextraction of acidic and basic compounds. Journal of Chromatography $A$, $1218,3945-3951$.

Neng, N. R., Silva, A. R. M., \& Nogueira, J. M. F. (2010). Adsorptive micro-extraction techniquesNovel analytical tools for trace levels of polar solutes in aqueous media. Journal of Chromatography A, 1217, 7303-7310.

Nojavan, S., \& Fakhari, A. R. (2010). Electro membrane extraction combined with capillary electrophoresis for the determination of amlodipine enantiomers in biological samples. Journal of Separation Science, 33, 3231-3238.

O'Reilly, J., Wang, Q., Setkova, L., Hutchinson, J. P., Chen, Y., Lord, H. L., et al. (2005). Automation of solid-phase microextraction. Journal of Separation Science, 28, 2010-2022.

Ouyang, G., \& Pawliszyn, J. (2006). Kinetic calibration for automated hollow fiber-protected liquid-phase microextraction. Analytical Chemistry, 78, 5783-5788.

Ouyang, G., Zhao, W., \& Pawliszyn, J. (2007). Automation and optimization of liquid-phase microextraction by gas chromatography. Journal of Chromatography A, 1138, 47-54.

Paleologos, E. K., Giokas, D. L., \& Karayannis, M. I. (2005). Micelle-mediated separation and cloud-point extraction. TrAC Trends in Analytical Chemistry, 24, 426-436.

Pedersen-Bjergaard, S., \& Rasmussen, K. E. (1999). Liquid-liquid-liquid microextraction for sample preparation of biological fluids prior to capillary electrophoresis. Analytical Chemistry, 71, 2650-2656.

Pedersen-Bjergaard, S., \& Rasmussen, K. E. (2006). Electrokinetic migration across artificial liquid membranes: New concept for rapid sample preparation of biological fluids. Journal of Chromatography A, 1109, 183-190.

Pena-Pereira, F., Costas-Mora, I., Lavilla, I., \& Bendicho, C. (2012). Rapid screening of polycyclic aromatic hydrocarbons (PAHs) in waters by directly suspended droplet microextractionmicrovolume fluorospectrometry. Talanta, 89, 217-222.

Pena-Pereira, F., Lavilla, I., Bendicho, C., Vidal, L., \& Canals, A. (2009). Speciation of mercury by ionic liquid-based single-drop microextraction combined with high-performance liquid chromatography-photodiode array detection. Talanta, 78, 537-541.

Pena, M. T., Casais, M. C., Mejuto, M. C., \& Cela, R. (2009). Development of an ionic liquid based dispersive liquid-liquid microextraction method for the analysis of polycyclic aromatic hydrocarbons in water samples. Journal of Chromatography A, 1216, 6356-6364. 
Petersen, N. J., Foss, S. T., Jensen, H., Hansen, S. H., Skonberg, C., Snakenborg, D., et al. (2010). On-chip electro membrane extraction with online ultraviolet and mass spectrometric detection. Analytical Chemistry, 83, 44-51.

Pezo, D., Salafranca, J., \& Nerín, C. (2007). Development of an automatic multiple dynamic hollow fibre liquid-phase microextraction procedure for specific migration analysis of new active food packagings containing essential oils. Journal of Chromatography A, 1174, 85-94.

Portugal, F. C. M., Pinto, M. L., \& Nogueira, J. M. F. (2008). Optimization of polyurethane foams for enhanced stir bar sorptive extraction of triazinic herbicides in water matrices. Talanta, 77 , 765-773.

Ramos Payán, M. D., Jensen, H., Petersen, N. J., Hansen, S. H., \& Pedersen-Bjergaard, S. (2012). Liquid-phase microextraction in a microfluidic-chip - High enrichment and sample clean-up from small sample volumes based on three-phase extraction. Analytica Chimica Acta, 735, 46-53.

Regueiro, J., Llompart, M., Garcia-Jares, C., Garcia-Monteagudo, J. C., \& Cela, R. (2008). Ultrasoundassisted emulsification-microextraction of emergent contaminants and pesticides in environmental waters. Journal of Chromatography A, 1190, 27-38.

Rezaee, M., Assadi, Y., Milani Hosseini, M.-R., Aghaee, E., Ahmadi, F., \& Berijani, S. (2006). Determination of organic compounds in water using dispersive liquid-liquid microextraction. Journal of Chromatography A, 1116, 1-9.

Romero, J., López, P., Rubio, C., Batlle, R., \& Nerín, C. (2007). Strategies for single-drop microextraction optimisation and validation: Application to the detection of potential antimicrobial agents. Journal of Chromatography A, 1166, 24-29.

Saleh, A., Yamini, Y., Faraji, M., Rezaee, M., \& Ghambarian, M. (2009). Ultrasound-assisted emulsification microextraction method based on applying low density organic solvents followed by gas chromatography analysis for the determination of polycyclic aromatic hydrocarbons in water samples. Journal of Chromatography A, 1216, 6673-6679.

Saraji, M., \& Bidgoli, A. (2010). Dispersive liquid-liquid microextraction using a surfactant as disperser agent. Analytical and Bioanalytical Chemistry, 397, 3107-3115.

Seidi, S., Yamini, Y., Saleh, A., \& Moradi, M. (2011). Electromembrane extraction of levamisole from human biological fluids. Journal of Separation Science, 34, 585-593.

Sharma, N., Jain, A., Singh, V. K., \& Verma, K. K. (2011). Solid-phase extraction combined with headspace single-drop microextraction of chlorophenols as their methyl ethers and analysis by high-performance liquid chromatography-diode array detection. Talanta, 83, 994-999.

Sikanen, T., Pedersen-Bjergaard, S., Jensen, H., Kostiainen, R., Rasmussen, K. E., \& Kotiaho, T. (2010). Implementation of droplet-membrane-droplet liquid-phase microextraction under stagnant conditions for lab-on-a-chip applications. Analytica Chimica Acta, 658, 133-140.

Silva, A. R. M., Portugal, F. C. M., \& Nogueira, J. M. F. (2008). Advances in stir bar sorptive extraction for the determination of acidic pharmaceuticals in environmental water matrices: Comparison between polyurethane and polydimethylsiloxane polymeric phases. Journal of Chromatography A, 1209, 10-16.

Sobhi, H. R., Yamini, Y., Esrafili, A., \& Abadi, R. H. H. B. (2008). Suitable conditions for liquid-phase microextraction using solidification of a floating drop for extraction of fat-soluble vitamins established using an orthogonal array experimental design. Journal of Chromatography $A$, 1196-1197, 28-32.

Tankeviciute, A., Kazlauskas, R., \& Vickackaite, V. (2001). Headspace extraction of alcohols into a single drop. The Analyst, 126, 1674-1677.

Turiel, E., \& Martín-Esteban, A. (2009). Molecularly imprinted polymers for solid-phase microextraction. Journal of Separation Science, 32, 3278-3284.

Vatinno, R., Vuckovic, D., Zambonin, C. G., \& Pawliszyn, J. (2008). Automated high-throughput method using solid-phase microextraction-liquid chromatography-tandem mass spectrometry 
for the determination of ochratoxin A in human urine. Journal of Chromatography A, 1201, 215-221.

Vidal, L., Chisvert, A., Canals, A., \& Salvador, A. (2007a). Sensitive determination of free benzophenone- 3 in human urine samples based on an ionic liquid as extractant phase in single-drop microextraction prior to liquid chromatography analysis. Journal of Chromatography A, 1174, 95-103.

Vidal, L., Domini, C. E., Grané, N., Psillakis, E., \& Canals, A. (2007b). Microwave-assisted headspace single-drop microextration of chlorobenzenes from water samples. Analytica Chimica Acta, 592, 9-15.

Vuckovic, D., Cudjoe, E., Hein, D., \& Pawliszyn, J. (2008). Automation of solid-phase microextraction in high-throughput format and applications to drug analysis. Analytical Chemistry, 80 , 6870-6880.

Vuckovic, D., Zhang, X., Cudjoe, E., \& Pawliszyn, J. (2010). Solid-phase microextraction in bioanalysis: New devices and directions. Journal of Chromatography A, 1217, 4041-4060.

Wang, H., \& Campiglia, A. D. (2008). Determination of polycyclic aromatic hydrocarbons in drinking water samples by solid-phase nanoextraction and high-performance liquid chromatography. Analytical Chemistry, 80, 8202-8209.

Wang, J.-X., Jiang, D.-Q., Gu, Z.-Y., \& Yan, X.-P. (2006). Multiwalled carbon nanotubes coated fibers for solid-phase microextraction of polybrominated diphenyl ethers in water and milk samples before gas chromatography with electron-capture detection. Journal of Chromatography A, 1137, 8-14.

Wang, Q., Qiu, H., Li, J., Liu, X., \& Jiang, S. (2010). On-line coupling of ionic liquid-based single-drop microextraction with capillary electrophoresis for sensitive detection of phenols. Journal of Chromatography A, 1217, 5434-5439.

Wanigasekara, E., Perera, S., Crank, J., Sidisky, L., Shirey, R., Berthod, A., \& Armstrong, D. (2010). Bonded ionic liquid polymeric material for solid-phase microextraction GC analysis. Analytical and Bioanalytical Chemistry, 396, 511-524.

Wu, H.-F., Yen, J.-H., \& Chin, C.-C. (2006). Combining drop-to-drop solvent microextraction with gas chromatography/mass spectrometry using electronic ionization and self-ion/molecule reaction method to determine methoxyacetophenone isomers in one drop of water. Analytical Chemistry, 78, 1707-1712.

Wu, J., \& Lee, H. K. (2005). Orthogonal array designs for the optimization of liquid-liquid-liquid microextraction of nonsteroidal anti-inflammatory drugs combined with high-performance liquid chromatography-ultraviolet detection. Journal of Chromatography A, 1092, 182-190.

Wu, J., \& Pawliszyn, J. (2001). Preparation and applications of polypyrrole films in solid-phase microextraction. Journal of Chromatography A, 909, 37-52.

Wu, Q., Chang, Q., Wu, C., Rao, H., Zeng, X., Wang, C., \& Wang, Z. (2010). Ultrasound-assisted surfactant-enhanced emulsification microextraction for the determination of carbamate pesticides in water samples by high performance liquid chromatography. Journal of Chromatography A, 1217, 1773-1778.

Wu, Y., Xia, L., Chen, R., \& Hu, B. (2008). Headspace single drop microextraction combined with HPLC for the determination of trace polycyclic aromatic hydrocarbons in environmental samples. Talanta, 74, 470-477.

Xu, H., Ding, Z., Lv, L., Song, D., \& Feng, Y.-Q. (2009). A novel dispersive liquid-liquid microextraction based on solidification of floating organic droplet method for determination of polycyclic aromatic hydrocarbons in aqueous samples. Analytica Chimica Acta, 636, 28-33.

Yang, Z.-H., Lu, Y.-L., Liu, Y., Wu, T., Zhou, Z.-Q., \& Liu, D.-H. (2011). Vortex-assisted surfactant-enhanced-emulsification liquid-liquid microextraction. Journal of Chromatography A, 1218, 7071-7077. 
Yangcheng, L., Quan, L., Guangsheng, L., \& Youyuan, D. (2006). Directly suspended droplet microextraction. Analytica Chimica Acta, 566, 259-264.

Yao, C., Pitner, W. R., \& Anderson, J. L. (2009). Ionic liquids containing the tris(pentafluoroethyl) trifluorophosphate anion: A new class of highly selective and ultra hydrophobic solvents for the extraction of polycyclic aromatic hydrocarbons using single drop microextraction. Analytical Chemistry, 81, 5054-5063.

Ye, C.-L., Zhou, Q.-X., \& Wang, X.-M. (2006). Headspace liquid-phase microextraction using ionic liquid as extractant for the preconcentration of dichlorodiphenyltrichloroethane and its metabolites at trace levels in water samples. Analytica Chimica Acta, 572, 165-171.

Ye, C., Zhou, Q., \& Wang, X. (2007). Improved single-drop microextraction for high sensitive analysis. Journal of Chromatography A, 1139, 7-13.

Yiantzi, E., Psillakis, E., Tyrovola, K., \& Kalogerakis, N. (2010). Vortex-assisted liquid-liquid microextraction of octylphenol, nonylphenol and bisphenol-A. Talanta, 80, 2057-2062.

Yin, X.-B. (2007). Dual-cloud point extraction as a preconcentration and clean-up technique for capillary electrophoresis speciation analysis of mercury. Journal of Chromatography A, 1154, 437-443.

Yu, C., Liu, Q., Lan, L., \& Hu, B. (2008). Comparison of dual solvent-stir bars microextraction and U-shaped hollow fiber-liquid phase microextraction for the analysis of Sudan dyes in food samples by high-performance liquid chromatography-ultraviolet/mass spectrometry. Journal of Chromatography A, 1188, 124-131.

Yu, J., Dong, L., Wu, C., Wu, L., \& Xing, J. (2002). Hydroxyfullerene as a novel coating for solid-phase microextraction fiber with sol-gel technology. Journal of Chromatography A, 978, 37-48.

Yu, L.-P. (2005). Cloud point extraction preconcentration prior to high-performance liquid chromatography coupled with cold vapor generation atomic fluorescence spectrometry for speciation analysis of mercury in fish samples. Journal of Agricultural and Food Chemistry, 53, 9656-9662.

Zeng, J., Chen, J., Song, X., Wang, Y., Ha, J., Chen, X., \& Wang, X. (2010). An electrochemically enhanced solid-phase microextraction approach based on a multi-walled carbon nanotubes/ Nafion composite coating. Journal of Chromatography A, 1217, 1735-1741.

Zeng, J., Yu, B., Chen, W., Lin, Z., Zhang, L., Lin, Z., et al. (2008). Application of ceramic/carbon composite as a novel coating for solid-phase microextraction. Journal of Chromatography A, 1188, 26-33.

Zeng, J., Zou, J., Song, X., Chen, J., Ji, J., Wang, B., et al. (2011). A new strategy for basic drug extraction in aqueous medium using electrochemically enhanced solid-phase microextraction. Journal of Chromatography A, 1218, 191-196.

Zhang, P.-P., Shi, Z.-G., Yu, Q.-W., \& Feng, Y.-Q. (2011). A new device for magnetic stirring-assisted dispersive liquid-liquid microextraction of UV filters in environmental water samples. Talanta, $83,1711-1715$.

Zhang, X., Oakes, K. D., Wang, S., Servos, M. R., Cui, S., Pawliszyn, J., et al. (2012). In vivo sampling of environmental organic contaminants in fish by solid-phase microextraction. TrAC Trends in Analytical Chemistry, 32, 31-39.

Zhang, Z., \& Pawliszyn, J. (1995). Quantitative extraction using an internally cooled solid phase microextraction device. Analytical Chemistry, 67, 34-43.

Zhao, F., Meng, Y., \& Anderson, J. L. (2008). Polymeric ionic liquids as selective coatings for the extraction of esters using solid-phase microextraction. Journal of Chromatography A, 1208, 1-9.

Zhou, Q., Bai, H., Xie, G., \& Xiao, J. (2008). Temperature-controlled ionic liquid dispersive liquid phase micro-extraction. Journal of Chromatography A, 1177, 43-49.

Zhu, F., Guo, J., Zeng, F., Fu, R., Wu, D., Luan, T., et al. (2010). Preparation and characterization of porous carbon material-coated solid-phase microextraction metal fibers. Journal of Chromatography $A, 1217,7848-7854$. 
Zhu, L., Zhu, L., \& Lee, H. K. (2001). Liquid-liquid-liquid microextraction of nitrophenols with a hollow fiber membrane prior to capillary liquid chromatography. Journal of Chromatography $A$, 924, 407-414.

Zhu, X., Cai, J., Yang, J., Su, Q., \& Gao, Y. (2006). Films coated with molecular imprinted polymers for the selective stir bar sorption extraction of monocrotophos. Journal of Chromatography A, 1131, 37-44. 